\title{
On Harder-Narasimhan filtration of the tangent bundle
}

\author{
INDRANIL BISWAS
}

\section{Introduction.}

Given a vector bundle $V$ on a polarized smooth projective variety, or more generally on a compact Kähler manifold, there is a natural filtration of $V$, called the Harder-Narasimhan filtration, such that the subsequent quotients are semi-stable sheaves satisfying a numerical condition. This filtration was introduced in [HN]. Here we are concerned with the HarderNarasimhan filtration of the tangent bundle.

If the canonical bundle is ample is then from a Theorem of Yau it follows that the tangent bundle is semi-stable with respect to the polarization $K$. In general, of course, the tangent bundle is not semi-stable. In Theorem 2.2 we give a criterion for the length of the Harder-Narasimhan filtration of the tangent bundle to be at most two.

There is a well-known question of whether any holomorphic bundle admitting a holomorphic connection actually admits a flat connection. In Theorem 3.1 we produce a class of compact Kähler manifolds with the property that any holomorphic bundle on them with a holomorphic connection admits a flat connection. The proofs of Theorem 2.2 and Theorem 3.1 are quite similar in spirit and involve, among other things, a systematic use of the Leibniz identity.

\section{A criterion for bounding the length.}

Let $X$ be an irreducible smooth projective variety over $\mathbb{C}$ of dimension $d$. Fix an ample line bundle $L$ on $X$.

For an $\mathcal{O}_{X}$ coherent sheaf $F$ on $X$, the degree of $X$ is defined by

$$
\operatorname{deg}(F):=\int_{X} c_{1}(F) \cup c_{1}(L)^{d-1} .
$$


For a torsion-free coherent sheaf $F$, the quotient $\operatorname{deg}(F) / \operatorname{rank}(F)$ is called the slope of $F$ and is denoted by $\mu(F)$.

Definition 2.1. A torsion-free $\mathcal{O}_{X}$ coherent sheaf $V$ on $X$ is called semistable (resp. stable) if for any proper sub-sheaf $0 \neq F \subset V$ with $V / F$ being torsion-free,

$$
\mu(F) \leq \mu(V) \quad(\text { resp. } \mu(F)<\mu(V)) .
$$

We will use the following convention. By a sub-sheaf of a torsion-free $\mathcal{O}_{X}$ coherent sheaf we will always mean a $\mathcal{O}_{X}$ coherent proper nonzero sub-sheaf such that the quotient is torsion-free. And by a quotient sheaf will mean a quotient by a sub-sheaf of the above type.

For any torsion-free coherent sheaf $V$ there is a unique filtration by subsheaves, called the Harder-Narasimhan filtration [Ko, Ch. V, Theorem 7.15]

$$
0=V_{0} \subset V_{1} \subset V_{2} \subset \ldots \subset V_{k-1} \subset V_{k}=V
$$

such that $V_{i} / V_{i-1}$ is the maximal semi-stable sub-sheaf of $V / V_{i-1}$. The integer $k$ will be called the length of the filtration. We will denote the number $\mu\left(V_{i} / V_{i-1}\right), 1 \leq i \leq k$, by $\mu_{i}(V)$.

Let $T_{0} \subset T_{1} \subset T_{2} \subset \ldots \subset T_{l}=T$ be the Harder-Narasimhan filtration of the tangent bundle $T$ of $X$.

Theorem 2.2. If the following three conditions are satisfied

(i) the first two sub-sheaves $T_{1}$ and $T_{2}$ are locally free;

(ii) $\mu_{1}(T):=\mu\left(T_{1}\right) \geq 0$;

(iii) The rank of the Neron-Severi group of $X$ is one, i.e.

$$
H^{1,1}(X) \cap H^{2}(X, \mathbb{Q})=\mathbb{Q}
$$

then either $T_{1}=T$ (i.e. $T$ is semi-stable) or $T_{2}=T$, i.e. $T$ is an extension of a semi-stable bundle by a semi-stable bundle with higher $\mu$.

Proof. For two local sections $s$ and $t$ of $T$, let $[s, t]$ denote their Lie bracket. We want to deduce from the condition (ii) above that $T_{1}$ is closed under Lie bracket. We will use the notation $\mathcal{T}_{1}$ to denote the vector bundle given by the sheaf $T_{1}$; similarly for $T_{2}$. Consider the following homomorphism of sheaves

$$
T_{1} \otimes_{\mathbb{C}} T_{1} \longrightarrow T / T_{1}
$$


which assigns to $s \otimes t$ the image of $[s, t]$ in $T / T_{1}$. The Leibniz formula for Lie bracket, namely

$$
[f s, t]=f \cdot[s, t]-<d f, t>. s
$$

and the identity $[X, Y]=-[Y, X]$ together imply that the homomorphism in (2.3) is $\mathcal{O}_{X}$-linear. In other words, it induces a homomorphism of bundles

$$
\psi: \mathcal{T}_{1} \otimes \mathcal{T}_{1} \longrightarrow T / \mathcal{T}_{1}
$$

The tensor product of two semi-stable sheaves is known to be semi-stable. Thus, since $\mathcal{T}_{1}$ is semi-stable, the tensor product $\mathcal{T}_{1} \otimes \mathcal{T}_{1}$ is also semi-stable. So, the $\mu$ of any quotient sheaf of $\mathcal{T}_{1} \otimes \mathcal{T}_{1}$ is greater than or equal to $\mu\left(\mathcal{T}_{1} \otimes \mathcal{T}_{1}\right)$. But

$$
\mu\left(\mathcal{T}_{1} \otimes \mathcal{T}_{1}\right)=2 . \mu\left(\mathcal{T}_{1}\right) \geq \mu\left(\mathcal{T}_{1}\right)
$$

The last inequality follows from the fact that $\mu\left(\mathcal{T}_{1}\right) \geq 0$. Since the $\mu$ of any sub-sheaf of $T / \mathcal{T}_{1}$ is less that or equal to $\mu_{2}(T)$ and $\mu_{2}(T)<\mu_{1}(T)$, considering the image of the homomorphism $\psi$ we conclude that the homomorphism $\psi$ must be zero. So $T_{1}$ is closed under Lie bracket. In other words, $\mathcal{T}_{1}$ is a holomorphic foliation on $X$. Now from the Theorem 3.2 and the subsequent identity (3.6) of $[\mathrm{L}]$ it follows that, for any integer $a>\operatorname{dim}_{\mathbb{C}}\left(T / \mathcal{T}_{1}\right)$, the characteristic class $\left(c_{1}\left(T / \mathcal{T}_{1}\right)\right)^{a}=0$. (Extend the partial connection along the foliation $\mathcal{T}_{1}$ given by the Lie-bracket on the normal bundle, $T / \mathcal{T}_{1}$, to a $G L(q, \mathbb{C})$ connection $\left(q=\operatorname{dim}_{\mathbb{C}}\left(T / \mathcal{T}_{1}\right)\right)$ on $T / \mathcal{T}_{1}$. Use this connection to calculate $c_{1}\left(T / \mathcal{T}_{1}\right)^{a}$ by Chern-Weil theory (as done in Theorem 3.2 of [L]). The identity (3.6) in [L] would imply that the Chern form for $\left(c_{1}\left(T / \mathcal{T}_{1}\right)\right)^{a}$, $a>\operatorname{dim}_{\mathbb{C}}\left(T / \mathcal{T}_{1}\right)$, vanishes identically. $)$

Since $H^{1,1}(X) \cap H^{2}(X, \mathbb{Q})=\mathbb{Q}$, the class $c_{1}\left(T / \mathcal{T}_{1}\right)$ is a scalar multiple of the polarization class. So $c_{1}\left(T / \mathcal{T}_{1}\right)^{a}=0$ implies that $c_{1}\left(T / \mathcal{T}_{1}\right)=0$. Since $T_{2} / T_{1}$ is the maximal semi-stable sub-sheaf of $T / \mathcal{T}_{1}$, if $\mathcal{T}_{2} \neq T$ then

$$
\mu_{2}(T)>0, \quad \text { and } \operatorname{deg}\left(T / \mathcal{T}_{2}\right)=\operatorname{deg}\left(T / T_{1}\right)-\operatorname{deg}\left(T_{2} / T_{1}\right)<0
$$

We want to show that $T_{2}$ in also closed under the Lie-bracket operation. This is obvious if $T_{2}=T$; assume that $T_{2} \neq T$. Using the homomorphism of sheaves, $\psi: T_{2} \otimes_{\mathbb{C}} T_{2} \longrightarrow T / T_{2}$, given by the Lie bracket, and the Leibniz rule together, we have a homomorphism of bundles $\psi: \mathcal{T}_{2} \otimes \mathcal{T}_{2} \longrightarrow T / \mathcal{T}_{2}$ as before.

Since $0 \longrightarrow \mathcal{T}_{1} \longrightarrow \mathcal{T}_{2}$ is the Harder-Narasimhan filtration of the bundle $\mathcal{T}_{2}$, the $\mu$ of any quotient of $\mathcal{T}_{2} \otimes \mathcal{T}_{2}$ is greater than or equal to $2 . \mu_{2}(T)$. The 
$\mu$ of any sub-sheaf of $T / \mathcal{T}_{2}$ is less that or equal to $\mu_{3}(T)$, and from the properties of the Harder-Narasimhan filtration we have

$$
\mu_{3}(T)<\mu_{2}(T)
$$

Since $\mu_{2}(T)>0$ (see (2.4)), we have $2 . \mu_{2}(T)>\mu_{2}(T)$. Thus from the above observations we get that the homomorphism $\psi$ must be zero. In other words, $T_{2}$ is closed under Lie-bracket. But, as earlier, that would imply that $\operatorname{deg}\left(T / T_{2}\right)=0$, which in turn would contradict (2.4). This completes the proof.

Remark 2.5. (i) The condition (ii) is satisfied, for example when $\operatorname{deg}(T) \geq$ 0 .

(ii) If $X$ is a smooth projective variety with ample canonical bundle then $X$ admits a Kähler-Einstein metric [Y]. This implies that $T$ is semi-stable with respect to the polarization $K_{X}$.

If the canonical bundle of $X$ is negative then there is an obstruction, known as the Futaki invariant, for the existence of Kähler-Einstein metric on $X[\mathrm{~F}]$.

\section{Bundles with holomorphic connections.}

In this section we assume $X$ to be a compact Kähler manifold equipped with a Kähler form $\omega$. As before, let

$$
T_{0} \subset T_{1} \subset T_{2} \subset \ldots \subset T_{l}=T
$$

be the Harder-Narasimhan filtration of the holomorphic tangent bundle of $X$.

We will use the following convention. For an holomorphic bundle $V$ on $X, \Omega^{i}(V)$ will denote the holomorphic bundle $V \otimes \Omega^{i}$; and $\Gamma^{i, j}(X, V)$ will denote the space of all $C^{\infty}(p, q)$-forms with values in $V$. We will not distinguish between a holomorphic bundle and the locally free $\mathcal{O}_{X}$-coherent sheaf corresponding to it.

A holomorphic structure on a $C^{\infty}$ bundle $V$ on $X$ is given by a first order operator $\bar{\partial}_{V}: \Gamma(X, V) \longrightarrow \Gamma^{0,1}(X, V)$ satisfying the Leibniz condition and the integrability condition $\bar{\partial}_{V}^{2}=0$. A holomorphic connection on a holomorphic bundle $V$ is a first order differential operator

$$
\partial: V \longrightarrow \Omega^{1}(V)
$$


satisfying the Leibniz condition $\partial(f . s)=d(f) . s+f \partial(s)$, where $f$ is a holomorphic function and $s$ is a (local) holomorphic section of $V$. It is easy to check that $\partial$ being a holomorphic connection is equivalent to the condition that the operator $\partial+\bar{\partial}_{V}$ is a connection with a holomorphic $\operatorname{End}(V)$ valued 2 -form as curvature.

Example 1. Let $\nabla$ be a flat connection on a $C^{\infty}$ bundle on $X$. Then the operator $\nabla^{0,1}$, the $(0,1)$ part of $\nabla$, gives a holomorphic structure on $V$, and $\nabla^{1,0}$ gives a holomorphic connection on the holomorphic bundle.

From the Chern-Weil construction of characteristic classes it is obvious that all the Chern classes of a bundle with holomorphic connection vanish.

A holomorphic vector bundle $V$ is said to admit a flat connection if there is a flat connection $\nabla$ on $V$ such that $\nabla^{0,1}=\bar{\partial}_{V}$.

Theorem 3.1. Let the Kähler manifold $X$ satisfy the condition that $\mu_{l}(T)$ $>0$. Let $V$ be a holomorphic bundle on $X$ admitting a holomorphic connection. Then $V$ admits a flat connection; also, the bundle $V$ is a direct sum of stable bundles of slope zero.

Proof. Let $V_{0} \subset V_{1} \subset V_{2} \subset \ldots \subset V_{n}=T$ be the Harder-Narasimhan filtration of the bundle $V$. Let $\partial$ be a holomorphic connection on $V$. Note that using the duality between $T$ and $\Omega^{1}, \partial$ induces a $\mathbb{C}$-linear homomorphism from the tensor product (over $\mathbb{C}$ ) of the sheaves, $T \otimes V$, to the sheaf $V$. Consider the following map of sheaves $T \otimes_{\mathbb{C}} V_{1} \longrightarrow V / V_{1}$ given by mapping $\theta \otimes s$ to the projection of $\partial_{\theta} s$ on $V / V_{1}$. Let $\mathcal{O}_{X}$ denote the sheaf of germs of holomorphic functions on $X$. The Leibniz condition implies that the above map is $\mathcal{O}_{X}$ linear, and hence it induces a homomorphism

$$
\psi: T \otimes_{\mathcal{O}_{X}} V_{1} \longrightarrow V / V_{1}
$$

Since $V_{1}$ is semi-stable and $\mu_{l}(T) \geq 0$, the $\mu$ of any quotient sheaf of $T \otimes_{\mathcal{O}_{X}} V_{1}$ is at least $\mu\left(V_{1}\right)$. This implies that the homomorphism $\psi$ is zero.

Since $\psi=0$, the holomorphic connection $\partial$ induces a holomorphic connection on $V_{1}$. Any $\mathcal{O}_{X}$ coherent sheaf with a holomorphic connection is locally free $[\mathrm{B}$, p. 211, Proposition 1.7]. (Though this proposition in [B] is stated for integrable connections ( $D$-modules), the proof uses only the Leibniz rule (in particular, does not use vanishing of curvature). The Leibniz rule is valid for a holomorphic connection.) 
Since the bundle $V_{1}$ admits a holomorphic connection, $c_{1}\left(V_{1}\right)=0$, and hence $\mu\left(V_{1}\right)=0$. Since $\mu(V)=0=\mu\left(V_{1}\right)$, we have $V=V_{1}$. This implies that $V$ is semi-stable.

If $V$ is actually a stable bundle then from [UY] it follows that $V$ admits a Hermitian-Yang-Mills connection; this metric is determined up-to a global scalar. We noted prior to Theorem 3.1 that the given condition that $V$ admits a holomorphic connection implies that all the Chern classes of $V$ vanish. This implies that the Hermitian-Yang-Mills connection is actually a flat unitary connection.

If $V$ is not stable then there is a filtration [K, Ch. $\mathrm{V}, \S 7$, Theorem 7.18]

$$
0=W_{0} \subset W_{1} \subset W_{2} \subset \ldots \subset W_{m-1} \subset W_{m}=V
$$

such that $W_{i} / W_{i-1}$ is a stable sheaf with $\mu\left(W_{i} / W_{i-1}\right)=\mu(V)$.

In view of the above mentioned result, [UY], that any stable vector bundle with vanishing Chern classes admits an unitary flat connection, Theorem 3.1 is implied by the following proposition.

Proposition 3.4. Each sub-sheaf $W_{i}, 1 \leq i \leq m$, in (3.3) is of the form $W_{i-1} \oplus U_{i}$, where $U_{i}$ is a stable bundle with vanishing Chern classes.

Proof. First we want to show that $W_{1}$ is a sub-bundle of $V$. In order to prove that we use the same method that has repeated been used in this paper : show that the holomorphic connection $\partial$ induces a holomorphic connection on $W_{1}$, and then using [B, p. 211, Proposition 1.7] (as used earlier in proving that $V$ is semi-stable) we may conclude that $W_{1}$ is locally free; we then show that $V / W_{1}$ has a quotient connection, and hence, using [B, p. 211, Proposition 1.7], $V / W_{1}$ is locally free. So $W_{1}$ must be a sub-bundle of $V$. We give the details of the argument below.

As in (3.2), the holomorphic connection $\partial$ induces a homomorphism of bundles $\phi: T \otimes W_{1} \longrightarrow V / W_{1}$. Since $W_{1}$ is semi-stable with $\mu\left(W_{1}\right)=0$, and $\mu_{l}(T)>0$, the $\mu$ of any quotient sheaf of $T \otimes W_{1}$ is strictly positive. This implies that the homomorphism $\phi$ is zero. So the connection $\partial$ induces a holomorphic connection on $W_{1}$. Let $\nu: V \rightarrow V / W_{1}$ denote the quotient projection. For a local section $s$ of $V / W_{1}$, consider $s \longmapsto \nu(\partial(\bar{s}))$, where $\bar{s}$ is a lift of $s$ to a local section of $V$. Since the sheaf $W_{1}$ is closed under the connection $\partial$, the section $\nu(\partial(\bar{s}))$ does not depend upon the choice of the lift $\bar{s}$ of $s$. Using this it is easy to see that the map $s \longmapsto \nu(\partial(\bar{s}))$ defines a holomorphic connection on $V / W_{1}$. So the sheaf $V / W_{1}$ is locally free. This implies that $W_{1}$ is a sub-bundle of $V$. As we already noted that the Chern 
classes of a bundle admitting a holomorphic connection vanish, since $W_{1}$ admits a holomorphic connection, all the Chern classes of $W_{1}$ vanish. Since $W_{1}$ is a stable bundle with vanishing Chern classes, from the Theorem of Uhlenbeck-Yau it admits a flat unitary structure.

Actually the same argument as above implies that any $W_{i}, 0 \leq i \leq m$, is a sub-bundle of $V$. To see this, first check that for any $W_{i}$, the analogue of $\phi$ is zero. So the connection $\partial$ induces a connection on $W_{i}$. Then the rest of the argument is identical.

Now we want to show that $W_{2}=W_{1} \oplus U_{2}$, where $U_{2}$ is a stable bundle with the property that all its Chern classes vanish. Since all the Chern classes of both $W_{1}$ and $W_{2}$ vanish, from the general properties of Chern classes it follows that the Chern classes of $W_{2} / W_{1}$ vanish. So, for a bundle $U_{2}$ satisfying the condition that $W_{2}=W_{1} \oplus U_{2}$, all the Chern classes of $U_{2}$ must vanish.

For notational simplicity we will denote $W_{2} / W_{1}$ by $W$. Note that since both $W_{1}$ and $W_{2}$ are sub-bundles of $V$, the sheaf $W$ is locally free. Let $c \in H^{1}\left(X, W^{*} \otimes W_{1}\right)$ be the element corresponding to the extension, namely $W_{2}$, of $W$ by $W_{1}$. Let $C^{0,1}\left(X, W^{*} \otimes W_{1}\right) \subset \Gamma^{0,1}\left(X, W^{*} \otimes W_{1}\right)$ be the space of all $\bar{\partial}$-closed $(0,1)$-forms with values in $W^{*} \otimes W_{1}$. Let $\rho: C^{0,1}\left(X, W^{*} \otimes\right.$ $\left.W_{1}\right) \longrightarrow H^{1}\left(X, W^{*} \otimes W_{1}\right)$ denote the surjection given by the Dolbeault resolution of $W^{*} \otimes W_{1}$. Define $I:=\rho^{-1}(c)$.

Since $W$ and $W_{1}$ are stable bundles with vanishing Chern classes, they admit flat unitary connections, namely the Hermitian-Yang-Mills connections. The Hermitian metrics on $W$ and and $W_{1}$ induce a Hermitian structure on $W^{*} \otimes W_{1}$. Let

$$
H \subset C^{0,1}\left(X, W^{*} \otimes W_{1}\right)
$$

be the subspace consisting of all harmonic forms. The map $\rho$ identifies $H$ with $H^{1}\left(X, W^{*} \otimes W_{1}\right)$. Consider $\hat{c}:=I \cap H$, the harmonic representative of the class $c$. Define the adjoint $\hat{c}^{*}$ of $\hat{c}$ as follows : Using the unitary structures on $W$ and $W_{1}$, for a (local) $C^{\infty}$ section $s$ of $W^{*} \otimes W_{1}=\operatorname{Hom}\left(W, W_{1}\right)$ we may consider its adjoint, $s^{*}$, which is a $C^{\infty}$ section of $\operatorname{Hom}\left(W_{1}, W\right)$, defined by $\left\langle s^{*}(a), b\right\rangle=\langle a, s(b)\rangle$, where $a$ is a local section of $W_{1}$ and $b$ is a local section of $W$. Now, if in some local holomorphic co-ordinate chart $\left(z_{1}, \ldots, z_{d}\right)$ on $X$ the section $\hat{c}$ is equal to $\sum_{k} \theta_{k} \otimes d \bar{z}_{k}$ then

$$
\hat{c}^{*}:=\sum_{k=1}^{d} \theta_{k}^{*} \otimes d z_{k}
$$


Since Hodge identities hold for Hermitian flat connections, the harmonicity of the section $\hat{c}$ implies that $\hat{c}^{*}$ is a holomorphic section of $\Omega^{1} \otimes \operatorname{Hom}\left(W_{1}, W\right)$. In other words,

$$
\hat{c}^{*} \in H^{0}\left(X, \operatorname{Hom}\left(T \otimes W_{1}, W\right)\right)
$$

But, since $\mu_{l}(T)>0$, there is no non-zero homomorphism from $T \otimes W_{1}$ to $W$ (both $W$ and $W_{1}$ are stable bundles with slope zero). So $\hat{c}^{*}=0$, which in turn implies that $\hat{c}=0$. Since the extension class vanishes, the exact sequence

$$
0 \longrightarrow W_{1} \longrightarrow W_{2} \longrightarrow W \longrightarrow 0
$$

splits. In other words, $W_{2}$ is isomorphic to $W_{1} \oplus W$.

Now we will use induction to complete the proof of the Proposition 3.4.

Assume that $W_{i}=W_{i-1} \oplus U_{i}$ for all $1 \leq i \leq j<m$ (as in the statement of the proposition). We want to show that $W_{j+1}=W_{j} \oplus U_{j+1}$, where $U_{j+1}$ is a stable bundle with vanishing Chern classes. From the assumption,

$$
W_{j}=\sum_{k=1}^{j} U_{k}
$$

where each $U_{k}$ is a stable bundle with vanishing Chern classes. Let $W$ denote the quotient $W_{j+1} / W_{j}$. Since both $W_{j+1}$ and $W_{j}$ are sub-bundles of $V$, the sheaf $W$ is locally free. Since the Chern classes of both $W_{j+1}$ and $W_{j}$ vanish (they admit holomorphic connections induced by $\partial$ ), the Chern classes of $W$ must also vanish. Corresponding to the short exact sequence of bundles

$$
0 \longrightarrow W_{j} \longrightarrow W_{j+1} \longrightarrow W:=W_{j+1} / W_{j} \longrightarrow 0
$$

we have the extension class $\xi \in H^{1}\left(X, \operatorname{Hom}\left(W, W_{j}\right)\right)$. Using the decomposition (3.6) we have

$$
\xi=\sum_{k=1}^{j} \xi_{k}
$$

where $\xi_{k} \in H^{1}\left(X, \operatorname{Hom}\left(W, U_{k}\right)\right)$. For any $1 \leq k \leq j$, both $W$ and $U_{k}$ are stable bundles with vanishing Chern classes. Recall the argument for proving the extension class $c$ corresponding to $W_{2}$ to be zero. Repeating this argument we get that the class $\xi_{k}$ must be zero for any $1 \leq k \leq j$. (In the argument for vanishing of the class $c, W_{1}$ should be replaced by $U_{k}$ to get the vanishing of $\xi_{k}$ ). So the extension class $\xi$ must vanish. This implies that the bundle $W_{j+1}$ is isomorphic to $W_{j} \oplus W$. This completes the proof of the Proposition 3.4. 
Remark 3.7. (i) The flat connection on $V$ that we obtained in Theorem 3.1 is actually an unitary flat connection.

(ii) Form the proof of Theorem 3.1 it follows that if $\mu_{l}(T) \geq 0$ and $V$ is a holomorphic bundle on $X$ with a holomorphic connection, then $V$ is semistable. The strict inequality, $\mu_{l}(T)>0$ in Theorem 3.1 was used to prove that $V$ is a direct sum of stable bundles of slope zero (which is a stronger statement than semi-stability).

(iii) Let $X$ be a compact Riemann surface of genus at least 2. Consider the flat $\operatorname{PSL}(2, \mathbb{R})$ connection on $X$ given by the uniformization theorem. This connection can be lifted to a flat $S L(2, \mathbb{R})$ connection, and the corresponding holomorphic bundle $V$ is given by the extension

$$
0 \longrightarrow K^{1 / 2} \longrightarrow V \longrightarrow K^{-1 / 2} \longrightarrow 0
$$

where $K^{1 / 2}$ is a square-root of the canonical bundle, and the extension class is $1 \in H^{1}\left(X, K^{1 / 2} \otimes K^{1 / 2}\right)=H^{1}(X, K)=\mathbb{C}$. This bundle $V$ is clearly not semi-stable. This example shows that in the statement of 3.7 (ii), the condition that $\mu_{l}(T) \geq 0$ is essential.

\section{References.}

[B] A. Borel et al. : Algebraic $D$-modules. Perspectives in Mathematics, Vol. 2 (Ed. J. Coates, S. Helgason), Academic Press.

[F] A. Futaki : An obstruction to the existence of Kähler-Einstein metrics. Inv. Math. 73 (1983) 437-443.

[HN] G. Harder, M. S. Narasimhan : On the cohomology groups of moduli spaces of vector bundles on curves. Math. Ann. 212 (1975) 215-248.

[Ko] S. Kobayashi : Differential geometry of complex vector bundles. Publications of Math. Soc. of Japan, Iwanami Schoten Pub. and Princeton University Press, 1987.

[L] H. B. Lawson : The quantitative theory of foliations. CBMS Regional Conference Series in Math. No. 27, AMS.

[NS] M. S. Narasimhan, C. Seshadri : Stable and unitary bundles on a compact Riemann surface. Ann. Math. 82 (1965) 540-564.

[UY] K. K. Uhlenbeck, S. T. Yau : On the existence of Hermitian-Yang-Mills connections in stable vector bundles. Comm. Pure Appl. Math. 39 (1986) 257-293. 
[Y] S. T. Yau : On the Ricci curvature of a compact Kähler manifold and the complex Monge-Ampère equation I. Comm. Pure Appl. Math. 31 (1978) 339-411.

School of Mathematics

TAta Institute of Fundamental Research

HOMI BHABHA ROAD, BOMBAY 400005, INDIA

INDRANIL@MATH.TIFR.RES.IN

RECEIVED MARCh 17Th, 1995. 